\title{
Gynaecologists' views on the management of Vaginal Vault Prolapse: A qualitative study
}

\author{
Omaema Al-Baghdadi ${ }^{1}$, Christian Barnick ${ }^{2}$, \\ Garima Srivastava ${ }^{3}$, Hassan M Elbiss ${ }^{4}$
}

\begin{abstract}
Objective: This study examined gynaecologists' experience and views on the management of vaginal vault prolapse (VVP) using laproscopic sarcocolpopexy (LSCP) versus open sarcocolpopexy (OSCP).

Methods: In a qualitative study conducted at the University of Surrey and Homerton University Hospital, UK, from 2016 to 2017, semi-structured interviews were conducted with 15 consultants experienced in minimal access surgery or urogynecology. Interviews were recorded and transcripts were analyzed using the qualitative description (QD) approach.

Results: Eight broad themes emerged: VVP management, LSCP for management of VVP, OSCP and vaginal surgery with or without mesh use in VVP management, laparoscopic training and support as well as surgeons' attitude towards LSCP. All participants acknowledged the importance of LSCP in the management of posthysterectomy VVP as benefits outweighed risks in their view. OSCP was considered suitable in very specific circumstances. Vaginal surgery could be an excellent alternative to OSCP bearing in mind long-term efficacy and sexual activity in young women. Most participants agreed with national recommendations to avoid use of mesh in vaginal surgery for VVP and expressed the view that it should be done in specialised centres by trained surgeons who do such operations.
\end{abstract}

Conclusions: This study showed that the acceptability of LSCP was dependent on participants' experience and consideration of the balance between patient's goals and potential risks. It provides useful guidance for future large-scale projects.

KEYWORDS: Vault prolapse, Qualitative research, Sacrocolpopexy, Laparoscopy.

How to cite this:

doi: https://doi.org/10.12669/pjms.38.3.5215

Al-Baghdadi O, Barnick C, Srivastava G, Elbiss HM. Gynaecologists' views on the management of Vaginal Vault Prolapse: $A$ qualitative study. Pak J Med Sci. 2022;38(3):583-588. doi: https://doi.org/10.12669/pjms.38.3.5215

This is an Open Access article distributed under the terms of the Creative Commons Attribution License (http://creativecommons.org/licenses/by/3.0), which permits unrestricted use, distribution, and reproduction in any medium, provided the original work is properly cited.

1. Omaema Al-Baghdadi,

Tawam Hospital, Al Ain, UAE.

Surrey University, Guildford, United Kingdom.

2. Christian Barnick,

3. Garima Srivastava,

4. Hassan M Elbiss,

Departments of Obstetrics and Gynaecology,

College of Medicine and Health Sciences,

UAE University, Al Ain, UAE.

2, 3: Homerton University Hospital,

Homerton Row, London, United Kingdom.

Correspondence:

Dr. Hassan Elbiss, MD, MRCOG, FRCOG. Department of Obstetrics and Gynaecology, College of Medicine and Health Sciences, Al-Ain, P.O. Box 17666, United Arab Emirates. E-mail: dr.hassan.elbiss@hotmail.com

* Received for Publication:

August 27, 2021

* $1^{\text {st }}$ Revision Received:

* $2^{\text {nd }}$ Revision Received:

* Final Revision Accepted:
September 6, 2021

September 29, 2021

October 20, 2021

\section{INTRODUCTION}

Genital prolapse, a common condition among women over 50 years of age, severely affects their life quality leading to withdrawal from social activity due to urinary, bowel and sexual dysfunction. ${ }^{1}$ Vaginal vault prolapse (VVP) is the main long-term problem following totalhysterectomy. ${ }^{2}$ The chance of undergoing surgery for prolapse is expected to increase with the continuing ageing of the population. ${ }^{3}$

The management of VVP involves pelvic floor physiotherapy, vaginal pessary and vaginal surgery including abdominal, vaginal and laparoscopic procedures to reinstate the normal anatomy including operations such as sacrospinous fixation, colpocliesis and sacrocolpopexy. ${ }^{4}$ The 
optimal surgical approach remains a considerable challenge and a controversy. Sacrocolpopexy, an abdominal surgical procedure for VVP that involves attaching a mesh between the vaginal vault and sacrum was first described as an open laparotomy. ${ }^{5}$ Open abdominal sacrocolpopexy (OSCP) has been viewed as the gold-standard procedure, ${ }^{6}$ being found to be superior to vaginal sacrospinous fixation. ${ }^{7}$ OSCP takes longer to perform, has a longer recovery time and is more expensive. ${ }^{7}$ Laparoscopic sacrocolpopexy (LSCP), first described in 1992, ${ }^{8}$ has evolved enormously with decreased morbidity, faster recovery and comparable outcomes. ${ }^{6,7}$

LSCP is gaining acceptance as the primary treatment for VVP. However, as longer time is required to train surgeons to be skilled in advancedlaparoscopy, gynaecologists are still cautious in using LSCP as an alternative to the traditional approach. Although surgical preferences and techniques for sacrocolpopexy worldwide have been reported to be similar. ${ }^{9}$ there are some geographical variations in LSCP and OSCP. ${ }^{6,7}$ It is surprising that none of the studies available have looked at the surgeons' values, opinions and clinical experience. Finding out from surgeons themselves how acceptable they find LSCP could help identify areas that could explain the lack of consensus about the preferred management of VVP. Difference in views and attitudes is best explored by qualitative research.

We investigated the reasons why gynaecologists are reluctant to adopt LSCP as the standard management of VVP despite the advancement of minimal-invasive approaches in gynaecology.

\section{METHODS}

After obtaining ethical and R\&D (FTFHMS-217-15, R\&D No 1653, 17 June 2016) approvals from the University of Surrey and Homerton University Hospital, UK, this qualitative study ${ }^{10,11}$ conducted in 2016-2017 used semi-structured interviews of participants experienced in post-hysterectomy VVP management in the UK. Infrmed consent was obtained from all participants. The interviews were conducted either face-to-face or via telephone. They were audio recorded and transcribed. Qualitative description (QD) method was used to gain an honest knowledge of professionals' experiences for tailoring clinical interventions via analysis of the interview transcripts to examine gynaecologists' acceptability of LSCP, their views about other surgical approaches (OSCP and vagi- nal surgery with or without mesh use) and the potential impact on their patients.

Participants were identified from regional and national professional society conferences. Overall, 18 consultant gynaecologists experienced in minimal-invasive procedures or uro-gynaecology were approached with the participant information sheet for making a voluntary contribution. All responded to the invitation to take part in the study $(100 \%)$. Consultant-level participants skilled in the management of VVP following total hysterectomy were included because it requires experience to advice women with VVP. Those with less than two-year experience at consultant level or with no experience in VVP management or minimal access surgery were excluded. Due to participant's availability and time constraints, 15 participants who were able to complete the interview were recruited into the study (83.3\%). All participants gave informed consent.

Sample size was according to the National Centre for Research Methods, which states: "a small number of cases or persons is extremely valuable and represent adequate numbers for a research project. This is accurate for studying hard to access people. Therefore, a relatively small number between six and twelve may be enough to give us insight" ${ }^{16}$ Interviews were started and continued until data became repetitive and the analysis reached saturation. Interviews were scheduled to coincide with the participant availability and permitted time. Wherever possible, interviews were conducted, face-to-face, in a private room; participants were given the option of a telephone interview if this was more suitable. This flexibility was considered necessary for avoiding bias. The confidentiality of participants in the study was preserved by keeping personal details and contact information separate from interview data in accordance with the UK Data Protection Act. Data were collected through semi-structured interviews to ensure high quality and genuine respondent data, and to minimize bias. Interviews were digitally recorded (Olympus VN-732PC) for transcription, ranging 15-54 min in duration.

An interview topic guide was designed based on published literature and expert opinion. It comprised of open-ended questions within a semi-structured format to explore gynaecologists' general experience and personal views of LSCP and their standard management of posthysterectomy VVP. This allowed the investigator to suggest the main topics for discussion and 
participants to offer additional comments. Then the interview progressed to ask specifically about the obstacles and expectations of LSCP as the standard management of post-hysterectomy VVP. Open-ended interview techniques were used to encourage comprehensive and diverse responses without the researcher's guidance. The interview agenda initially consisted of the following board areas: general information, experience of LSCP, OSCP, vaginal surgery with or without mesh in VVP, views towards training in laparoscopy and LSCP, acceptability of LSCP from their own perspective; cost, unit and work environment, and attitudes towards LSCP.

The recorded and transcribed data were interpreted by two researchers (a consultant gynaecologist and research-design expert). The QD approach explored gynaecologists' experience and acceptability of LSCP for VVP management with respect to "why", "how" and "what" questions about views and worries. Therefore, with its mainly inductive approach qualitative analysis was used for problem identification, theory formation and concept development. Commencing after the first five interviews, the interview data were organized and coded into themes manually, which involved identifying and highlighting emergent themes that could be considered particularly important to the interviewee. This process allowed the development of a working analytical framework where thematic codes were grouped together into categories for use in subsequent transcripts. This required judgment in retaining the original meanings and 'feel' of the interviewees' words. For internal reliability a third researcher independently sampled and reassessed the QD analyses carried out.

\section{RESULTS}

Of the total of 15 interviews conducted, nine were face-to-face and six were via telephone. There were eleven males $(73.3 \%)$. The mean age was 55.86 years (range 39-65). Three were retired consultants. The duration of experience was on average 14.4 years (range 2-25). Five of the interviewed consultants were laparoscopic surgeons, five were uro-gynaecologists and five both. All surgeons were trained in the UK and 12 $(80 \%)$ had attended short term courses in Europe, Asia and USA. All participants had laparoscopic experience and their practice of minimal access surgery (MAS) was on average $64.3 \%$ of their surgical work (range 20-90\%). Regarding LSCP, three consultants didn't do the procedure (two stopped after a period of use), $12(80 \%)$ did it regularly with annual average of ten cases (range 4-40). About OSCP, eleven (73.3\%) consultants didn't do the open surgery; only four (26.6\%) did it on a regular basis with an average of seven cases annually (range 4-10). Vaginal surgery was done by all consultants but five didn't do it for VVP; annual average 47.5 cases of SSF (range $5-150)$. Mesh use vaginally was only deployed by three $(20 \%)$ (two used modified tapes and one bio-meshes) in around 5-50 cases per year. Eight board themes and sub-themes that emerged from analysis of all interviews are shown in Table-I.

These wide-ranging themes were identified using QD till data saturation. Participants acknowledged the importance of LSCP in the management of post-hysterectomy VVP as benefits outweighed the risks in their view. While LSCP was considered an "excellent approach" and "standard" by twelve surgeons; three participants considered it complex and thought it was not a safe procedure to offer it as first line. All participants considered OSCP to be a highly morbid procedure that was a good option for women with severe symptomatic VVP and recurrent prolapse as well as in situations when surgeons need to convert to laparotomy following failed LSCP. Vaginal approach was considered the "general choice" in VVP management. LSCP as a treatment of VVP was evaluated for benefits and limitations, barriers to success, the future of surgery and the available research. Concerning benefits associated with LSCP, all agreed that it had quick recovery, short hospital state, and value for young fit sexually active women.

Six participants appraised the good visualization, especially in difficult cases with high BMI and intra-abdominal adhesions. Most of the interviewees agreed on the fact that it was "wrong to give ladies serious morbidity for something that is a QoL issue". They considered women with previous surgeries and potential intra-abdominal adhesions, high BMI, not fit for MAS, anaesthesia risk, and serious medical co-morbidities as highrisk women for an abdominal approach. Other group of surgeons considered high BMI women and abdominal adhesions as better for LSCP. With respect to evidence, participants clarified the lack of comparative studies: "Patients are difficult to match. It's difficult to get large numbers unless you do multicenter studies. If you do multicenter studies, you are looking at lots of different surgeons. Different surgeons have slightly different techniques and even if you standardize 
Table-I: Themes and subthemes from interviews conducted in a qualitative study concerning gynaecologists' views on the management of vaginal vault prolapse.

\begin{tabular}{|c|c|}
\hline Main theme & Sub theme \\
\hline \multirow{3}{*}{ Vulvovaginal prolapse management } & Surgeon's treatment of choice \\
\hline & Patient selection \\
\hline & Quality of life \\
\hline \multirow{7}{*}{ Laproscopic sacrocolpopexy for management of vulvovaginal prolapse } & Surgeon's opinion \\
\hline & Surgeon's treatment of choice \\
\hline & Patient selection surgery \\
\hline & Benefits of surgery \\
\hline & Limitations of surgery \\
\hline & Surgical Training \\
\hline & Evidence based medicine \\
\hline \multirow{4}{*}{ Open sacrocolpopexy for management of vulvovaginal prolapse } & Surgeon's opinion \\
\hline & Patient selection for surgery \\
\hline & Benefits of surgery \\
\hline & Limitations of surgery \\
\hline \multirow{4}{*}{ Vaginal surgery for management of vulvovaginal prolapse } & Surgeon's opinion \\
\hline & Patient selection for surgery \\
\hline & Benefits of surgery \\
\hline & Limitations of surgery \\
\hline \multirow{5}{*}{ Mesh use in management of vulvovaginal prolapse } & Surgeon's opinion \\
\hline & Benefits of mesh use \\
\hline & Limitations of mesh use \\
\hline & Patient's judgement \\
\hline & Patient's counselling \\
\hline \multirow{4}{*}{ Training in the United Kingdom } & Surgeon's opinion \\
\hline & Surgeon's learning curve \\
\hline & Situation of training \\
\hline & Limitations to training \\
\hline \multirow{3}{*}{ Support to do laproscopic sacrocolpopexy } & Work culture \\
\hline & Cost factor \\
\hline & Equipment and technology \\
\hline \multirow{3}{*}{ Surgeons' attitude towards laproscopic sacrocolpopexy } & Surgeon's background \\
\hline & Lack of agreement \\
\hline & Stimulating though \\
\hline
\end{tabular}

the techniques, they have different levels of ability and different types of procedure, whether it's laparoscopic or vaginal."

Advantages and disadvantages of mesh use in the management of VVP abdominally (LSCP/ OSCP) or vaginally was considered critical inpatient counselling. Medicolegal issues related to mesh use was conveyed with recommendations to refer patients to tertiary centers. Participants' vision of training in laparoscopy and LSCP in the UK shed light on the learning process as well as the future of training and the limitations faced.
All surgeons believed it to be the main barrier to the evolution of LSCP in the UK. Most agreed training was self-motivated that needed hands-on learning first based in wet-labs and animals, and then finally in humans. LSCP was an advanced procedure that needed advanced skills, which meant that it need time to learn, appropriate theatres and equipment besides good trainer and assistant. Participants also opined critically regarding workplace, support and finance, and their impact on MAS and access to LSCP in the UK. 


\section{DISCUSSION}

The demand for treatment of VVP, a longterm problem following total-hysterectomy, has increased in the recent years due to ageing population. It is a well-known condition including in low-middle income countries. ${ }^{12-15}$ This research explored experienced gynaecologists' opinions and acceptability of LSCP as the standard option in the management of post-hysterectomy VVP. Published research and studies in the literature largely focus on outcomes and modalities rather than examining surgeons' perspectives. To our knowledge there was no qualitative research about the standard approach to manage women with VVP at the time of undertaking the work. This study obtained original data exploring surgeons' expectations and responses to information on LSCP and other approaches to VVP management. It considered whether LSCP approach influenced VVP management by exploring participants' views about the laparoscopic approach compared to others approaches, and questioned whether additional areas of targeted training could benefit surgeons to practice such approaches in the future.

Undertaking the QD approach allowed themes to emerge and gave the opportunity to explore concepts, opinions and experiences from collected data. Most of the themes resonated with published quantitative research. Thus, qualitative research enriched the evidence base through the explanation of the professional and personal impact of LSCP approach and other methods. Using semi-structured interviews was an effective means of identifying issues important to surgeons, supported by a sample size adequate for valid qualitative results. Our manuscript complied with a majority of the items of the COREQ reporting checklist. $^{16}$ Being the first qualitative study examining surgeons view about a novel issue, our findings merit consideration.

Limitations: One limitation was interviewee time constraint. The duration of experience of the participants was variable, a feature that adds to the spectrum of our observations. The consultants were mostly experienced in laparoscopic procedures, so possibly their attitudes were biased in favor of this technique over other approaches. Additionally, as participants were selected from scientific courses, this may impact on generalisability of findings. Another limitation is the disproportionately low female consultant participation $(4,26.6 \%)$. A final limitation of the study could be that by taking part in the study the involved surgeons had already expressed their acceptance of such surgery and they wanted to validate their views. Eliciting data through semi-structured interviews relies heavily on the rapport between researcher and participant. The surgeons were identified by the researcher and this combined with the satisfactory sample size and the in-depth analysis produced findings descriptive of a wider sector of gynaecologists.

LSCP's recent popularity may be related to the decrease in use of mesh vaginally. ${ }^{17}$ There has been a decrease in mesh use vaginally from $25 \%$ to $2 \%{ }^{18}$ As a treatment of choice, some consider LSCP to be better than vaginal approach especially in young sexually active women, but this view was not universal in our study. Although LSCP and OSCP have similar outcomes, ${ }^{19}$ more than half the participants considered LSCP as a better option and one rated OSCP higher. The current opposition among some gynaecologists was likely because they were still cautious to use it due to the lack of advanced-laparoscopy skills. One of the published studies reported on the lack of structured curricula; inadequate faculty expertise, and limited time were the most important obstacles. ${ }^{20}$ Evidently, most of the published reports on LSCP were small retrospective and comparative-cohort studies. ${ }^{21,22}$ Application and maintenance of an advanced surgical technique is a challenge to practicing consultants and their patients. Future research, considering the importance of patient and public engagement, ${ }^{23}$ would also benefit from evaluating women's experience of the LSCP approach.

\section{CONCLUSION}

Implementation of a new surgical technique is an ever-present challenge to practicing surgeons and their patients. Laparoscopy is one of these innovative technologies that offers several potential benefits above traditional open or vaginal procedures. This first qualitative study provides new information concerning gynaecologists' views on the management of VVP and LSCP as the standard approach in this situation. The clinical significance of our findings are that LSCP was recognized as the standard "first line" management of VVP when SCP was indicated. It was viewed as having great value for young sexually active women and as having a high satisfaction rate among patients.

Competing interests: None.

Grant Support and Financial Disclosures: None. 


\section{REFERENCES}

1. Hendrix SL, Clark A, Nygaard I, Aragaki A, Barnabei V, McTiernan A. Pelvic organ prolapse in the Women's Health Initiative: gravity and gravidity. Am J Obstet Gynecol. 2002;186(6):1160-1166. doi: 10.1067/mob.2002.123819

2. RosatiM,BramanteS, BracaleU,PignataG,AzioniG.Efficacy of laparoscopic sacrocervicopexy for apical support of pelvic organ prolapse. J Soc Laparo Robotic Surg. 2013;17(2):235244. doi: $10.4293 / 108680813 X 13654754535115$

3. Akyol A, Akca A, Ulker V, Gedikbasi A, Kublay A, Han A, et al. Additional surgical risk factors and patient characteristics for mesh erosion after abdominal sacrocolpopexy. J Obstet Gynaecol Res. 2014;40(5):13681374. doi: $10.1111 /$ jog. 12363

4. Vandendriessche D, Sussfeld J, Giraudet G, Lucot JP, Behal H, Cosson M. Complications and reoperations after laparoscopic sacrocolpopexy with a mean follow-up of 4 years. Int Urogyn J. 2017;28(2):231-239. doi: 10.1007/ s00192-016-3093-6

5. Arthure HG, Savage D. Uterine prolapse and prolapse of the vaginal vault treated by sacral hysteropexy. J Obstet Gynaecol Br Emp. 1957;64(3):355-360. doi: 10.1111/j.14710528.1957.tb02652.x

6. Murphy AM, Clark CB, Denisenko AA, D'Amico MJ, Vasavada SP. Surgical management of vaginal prolapse: current surgical concepts. Can J Urol. 2021;28(S2):22-26.

7. Dieter AA. Pelvic Organ Prolapse: Controversies in Surgical Treatment. Obstet Gynecol Clin North Am. 2021;48(3):437-448. doi: 10.1016/j.ogc.2021.05.001

8. Ostrzenski A. Laser video-laparoscopic colpopexy. Ginekol Pol. 1992;63(7):317-323.

9. Bassaly R, McCullough M, Hussamy D, Downes K, Hoyte L, Hart $S$. Technical preferences of surgeons performing a sacrocolpopexy procedure. Surg Tech Int. 2012;22:189-194.

10. Baker SE, Edwards R. How many qualitative interviews is enough? Expert voices and early career reflections on sampling and cases in qualitative research. Southampton: National Centre for Research Methods. 2012.

11. Gibbs G. Qualitative data analysis: explorations with NVivo. London: Open University Press. 2002.

12. Jokhio AH, Rizvi RM, MacArthur C. Prevalence of pelvic organ prolapse in women, associated factors and impact on quality of life in rural Pakistan: population-based study. BMC Womens Health. 2020;20:82. doi: 10.1186/ s12905-020-00934-6

13. Kayondo M, Kaye DK, Migisha R, Tugume R, Kato PK, Lugobe HM, Geissbuehler V. Impact of surgery on quality of life of Ugandan women with symptomatic pelvic organ prolapse: a prospective cohort study. BMC Womens Health. 2021;21(1):258. doi: 10.1186/s12905-021-01397-z

14. Prateek S, Mundhra R, Bahadur A, Kunwar K, Jha P, Chawla L. Women's experiences and perception of symptomatic pelvis organ prolapse: A Cross sectional study from Uttarakhand, India. J Family Med Prim Care. 2021;10(1):449-453. doi: 10.4103/jfmpc.jfmpc_1763_20
15. Gedefaw G, Demis A. Burden of pelvic organ prolapse in Ethiopia: a systematic review and meta-analysis. BMC Womens Health. 2020;20(1):166. doi: 10.1186/s12905-02001039-w

16. Tong A, Sainsbury P, Craig J. Consolidated criteria for reporting qualitative research (COREQ): a 32-item checklist for interviews and focus groups. Int J Qual Health Care. 2007;19(6):349-357. doi: 10.1093/intqhc/mzm042

17. Chevrot A, Droupy S, Linares E, de Tayrac R, Costa P, Wagner L. Impact of laparoscopic sacrocolpopexy on symptoms, health-related quality of life and sexuality: A 3-year prospective study. Prog Urol. 2016;26(10):558-565. doi: 10.1016/j.purol.2016.02.009

18. Takacs EB, Kreder KJ. Sacrocolpopexy: Surgical Technique, Outcomes, and Complications. Current Urol Rep. 2016;17(12):90. doi: 10.1007/s11934-016-0643-x

19. Maher C, Baessler K, Glazener CM, Adams EJ, Hagen S. Surgical management of pelvic organ prolapse in women. Cochrane Database Syst Rev. 2007(3):CD004014. doi: 10.1002/14651858.CD004014.pub5

20. Byrne J, Straub H, DiGiovanni L, Chor J. Evaluation of ethics education in obstetrics and gynecology residency programs. Am J Obstet Gynecol. 2015;212(3):397. e1-8. doi: 10.1016/j.ajog.2014.12.027

21. Marcickiewicz J, Kjollesdal M, Engh ME, Eklind S, Axen C, Brannstrom M, et al. Vaginal sacrospinous colpopexy and laparoscopic sacral colpopexy for vaginal vault prolapse. Acta Obstet Gynecol Scand. 2007;86(6):733-738. doi: 10.1080/00016340701332811

22. Seracchioli R, Hourcabie JA, Vianello F, Govoni F, Pollastri P, Venturoli S. Laparoscopic treatment of pelvic floor defects in women of reproductive age. J Am Assoc Gynecol Laparosc. 2004;11(3):332-335. doi: 10.1016/S10743804(05)60045-X

23. Moss N, Daru J, Lanz D, Thangaratinam S, Khan KS. Involving pregnant women, mothers and members of the public to improve the quality of women's health research. BJOG. 2017;124(3):362-365. doi:10.1111/1471-0528.14419

\section{Authors' Contribution:}

OAB and HME conceived and designed the study and performed analysis and editing of manuscript and both are responsible and accountable for the accuracy or integrity of the work.

CB and GS contributed to data collection and manuscript writing, and all authors did review and final approval of the manuscript. 